\title{
The isoscalar monopole resonance of the alpha particle: a prism to nuclear Hamiltonians
}

\author{
Sonia Bacca ${ }^{1}$, Nir Barnea ${ }^{2}$ Winfried Leidemann ${ }^{3,4}$, Giuseppina Orlandini ${ }^{3,4}$ \\ ${ }^{1}$ TRIUMF, 4004 Wesbrook Mall, Vancouver, B.C. V6J 2A3, Canada \\ ${ }^{2}$ Racah Institute of Physics, The Hebrew University, 91904, Jerusalem, Israel \\ ${ }^{3}$ Dipartimento di Fisica, Università di Trento, I-38123 Trento, Italy \\ ${ }^{4}$ Istituto Nazionale di Fisica Nucleare, Gruppo Collegato di Trento, I-38123 Trento, Italy
}

\begin{abstract}
We present an ab-initio study of the isoscalar monopole excitations of ${ }^{4} \mathrm{He}$ using different realistic nuclear interactions, including modern effective field theory potentials. In particular we concentrate on the transition form factor $F_{\mathcal{M}}$ to the narrow $0^{+}$resonance close to threshold. $F_{\mathcal{M}}$ exhibits a strong potential model dependence, and can serve as a kind of prism to distinguish among different nuclear force models. Comparing to the measurements obtained from inelastic electron scattering off ${ }^{4} \mathrm{He}$, one finds that the state-of-the-art theoretical transition form factors are at variance with experimental data, especially in the case of effective field theory potentials. We discuss some possible reasons for such discrepancy, which still remains a puzzle.
\end{abstract}

The isoscalar monopole strength of large nuclei has been extensively studied since the discovery of a giant monopole resonance in ${ }^{144} \mathrm{Sm}$ and ${ }^{208} \mathrm{~Pb}[1]$. The reason for the great interest in such excitations originates from their connection to the incompressibility modulus of infinite nuclear matter [2, 3]. The alpha particle is a light nucleus, that however has a binding energy per particle similar to that of large systems and a high central density. While it possesses no bound excited states, it exhibits a very pronounced narrow resonance $\left({ }^{4} \mathrm{He}^{*}\right)$ with the same quantum numbers $0^{+}$as the ground state, i.e., an isoscalar monopole resonance. Today, the development of few-body theories has reached a point, where an ab-initio calculation of the four-body isoscalar monopole transition strength can be carried out with high precision. As will become evident in the following, the comparison of such four-body results with experimental data can serve as a stringent test for nuclear Hamiltonians, that are the sole ingredients of an ab-initio quantum mechanical approach.

The four-nucleon isoscalar monopole resonance is located at $E_{R}^{e x p}=-8.20 \pm 0.05 \mathrm{MeV}$, with a width of $270 \pm 50 \mathrm{keV}$ [4], just above the first two-body break-up threshold $E_{t h r}^{p}=-8.48 \mathrm{MeV}$ into a proton and a triton and below the next threshold $E_{t h r}^{n}=-7.74 \mathrm{MeV}$ into a neutron and a ${ }^{3} \mathrm{He}$. A summary about the experimental studies of the spectrum of ${ }^{4} \mathrm{He}$ can be found in Ref. [5]. Valuable information about the nature of the resonance is given by the transition form factor $F_{\mathcal{M}}(q)$ measured in electron scattering experiments $\left({ }^{4} \mathrm{He}\left(e, e^{\prime}\right)^{4} \mathrm{He}^{*}\right)$ at various momentum transfer $q$. Similarly to the case of the elastic form factor, the $q$ dependence of $F_{\mathcal{M}}$ reflects the dynamics at various interaction ranges.

The progress in ab-initio few-body methods allows today to obtain accurate results for observables in light nuclear systems using realistic potential models (see review [6]). In recent years the debate regarding potential models has boosted, especially after the introduction of the effective field theory (EFT) strategy in nuclear physics [7]. At present, both phenomenological realistic and chiral EFT potentials are used in ab-initio calculations, but only for very few observables large differences are found, e.g., for the polarization observable $A_{y}$ of $p$ ${ }^{3} \mathrm{He}$ scattering [8]. In this letter, we point out that the calculated ${ }^{4} \mathrm{He}$ isoscalar monopole resonance transition form factor $F_{\mathcal{M}}(q)$ depends dramatically on the nuclear Hamiltonian. Thus, it can serve as a kind of prism to distinguish among nuclear force models.

Main Results. The isoscalar monopole strength $S_{\mathcal{M}}(q, \omega)$ is in general a function of $q$ and the energy transfer $\omega$. It is given by

$$
\begin{aligned}
& S_{\mathcal{M}}(q, \omega)=\sum d n|\langle n|\mathcal{M}(q)| 0\rangle|^{2} \delta\left(\omega-E_{n}+E_{0}\right) \\
= & -\frac{1}{\pi} \operatorname{Im}\left\langle 0\left|\mathcal{M}^{\dagger}(q) \frac{1}{\omega-H+E_{0}+i \epsilon} \mathcal{M}(q)\right| 0\right\rangle
\end{aligned}
$$

where $|0\rangle,|n\rangle$ and $E_{0}, E_{n}$ are eigenfunctions and eigenvalues of the nuclear Hamiltonian $H$, and

$$
\mathcal{M}(q)=\frac{G_{E}^{s}(q)}{2} \sum_{i}^{A} j_{0}\left(q r_{i}\right)
$$

is the isoscalar monopole operator. Here $G_{E}^{s}(q)=$ $G_{E}^{p}(q)+G_{E}^{n}(q)$ is the nucleon electric isoscalar form factor [9], $\boldsymbol{r}_{i}$ is the nucleon's position, and $j_{0}$ is the spherical Bessel function of $0^{t h}$ order. The monopole strength can be written as a sum of a resonance term $S_{\mathcal{M}}^{\text {res }}$ and a nonresonant background contribution $S_{\mathcal{M}}^{\mathrm{bg}}$,

$$
S_{\mathcal{M}}(q, \omega)=S_{\mathcal{M}}^{\mathrm{res}}(q, \omega)+S_{\mathcal{M}}^{\mathrm{bg}}(q, \omega) .
$$

For a narrow resonance one defines the resonance transition form factor

$$
\left|F_{\mathcal{M}}(q)\right|^{2}=\frac{1}{Z^{2}} \int d \omega S_{\mathcal{M}}^{\mathrm{res}}(q, \omega) .
$$

In Fig. 1] we show results for $F_{\mathcal{M}}(q)$ with two different Hamiltonians including realistic three-nucleon forces $(3 \mathrm{NF})$ in comparison to experimental data from inelastic electron scattering [4, 11, 12]. As Hamiltonians we 


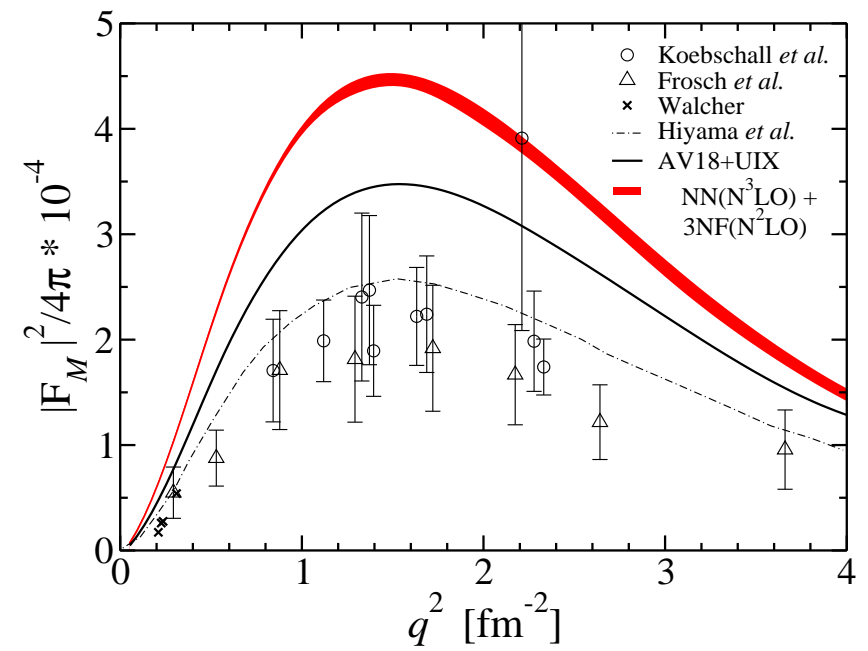

FIG. 1. (Color online) Theoretical transition form factor $\left|F_{\mathcal{M}}\left(q^{2}\right)\right|^{2}$ with $G_{E}^{n}=0$ calculated with various force models: AV18+UIX (full line), $\mathrm{N}^{3} \mathrm{LO}+\mathrm{N}^{2} \mathrm{LO}$ (red band); result from [10] (dot-dashed). Data from Frosch et al. [11], Walcher [4] and Köbschall et al. [12].

use (i) the AV18 [13] NN potential plus the UIX [14] $3 \mathrm{NF}$, (ii) an EFT based potential, where we take the NN potential 15] at fourth order $\left(\mathrm{N}^{3} \mathrm{LO}\right)$ in the chiral expansion augmented by a $3 \mathrm{NF}$ at order $\mathrm{N}^{2} \mathrm{LO}$ [16]. The Coulomb potential is taken into account in all calculations. Both the EFT and the AV18 NN potentials reproduce the NN scattering phase shifts with high precision $\left(\chi^{2} /\right.$ datum $\left.\approx 1\right)$. In the EFT calculations, two different parameterizations of the $3 \mathrm{NF}$ have been used, leading to the red band in Fig. 1, The chiral low energy constants $c_{D}$ and $c_{E}$ have been determined either by setting $c_{D}$ to a reasonable value and then fitting $c_{E}$ to the threenucleon binding energies [16] $\left(c_{D}=1\right.$ and $\left.c_{E}=-0.029\right)$ or by fitting to the ${ }^{3} \mathrm{H}$ binding energy and beta decay [17] $\left(c_{D}=-0.2\right.$ and $\left.c_{E}=-0.205\right)$. We also display the result of a previous calculation by Hiyama et al. [10], with the AV8' potential, a reduced version of AV18, and a simplified central 3NF, fitted to the binding energy of ${ }^{3} \mathrm{H}$. All three Hamiltonians reproduce the ${ }^{4} \mathrm{He}$ experimental binding energy within one percent. Surprisingly, the results for $F_{\mathcal{M}}(q)$ strongly depend on the Hamiltonian. Furthermore, the realistic Hamiltonians fail to reproduce the experimental data. In particular, this is true for the EFT forces that predict a transition form factor twice as large as the measured one.

In contrast, the realistic Hamiltonians lead to rather similar results for the elastic form factor $F_{e l}(q)$ of ${ }^{4} \mathrm{He}$, defined as

$$
F_{\mathrm{el}}(q)=\frac{1}{Z}\langle 0|\mathcal{M}(q)| 0\rangle .
$$

In Fig. 2, $F_{e l}(q)$ is shown for the AV18+UIX model and for the chiral EFT potentials. The fact that the results do not differ significantly is not very surprising, since both Hamiltonians give a very similar result for the ra-

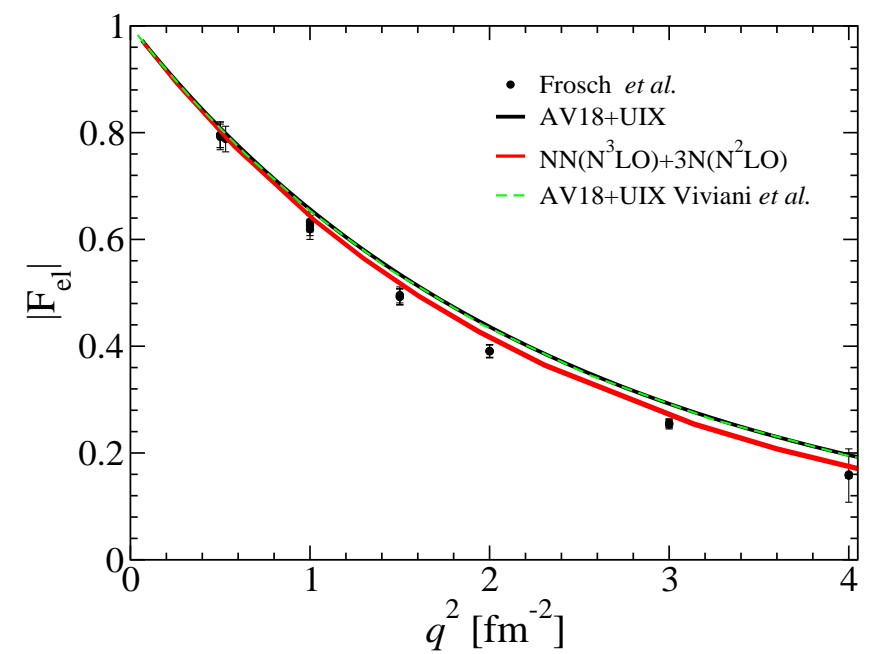

FIG. 2. (Color online) Elastic form factor $\left|F_{e l}\left(q^{2}\right)\right|$ of ${ }^{4} \mathrm{He}$ calculated with various force models: AV18+UIX (full line); $\mathrm{N}^{3} \mathrm{LO}+\mathrm{N}^{2} \mathrm{LO}$ (red band); result from 18] with AV18+UIX (dot-dashed). Data from Frosch et al. [19].

TABLE I. Ground state energies in $\mathrm{MeV}$ for ${ }^{3} \mathrm{H},{ }^{3} \mathrm{He}$ and ${ }^{4} \mathrm{He}$ with $\mathrm{N}^{3} \mathrm{LO}$ [15] and $\mathrm{N}^{2} \mathrm{LO}$ (parameterizations from 16] or [17]). Comparison of present results (EIHH) with no core shell model (NCSM) and hyperspherical harmonics (HH) results.

\begin{tabular}{ccccc}
\hline \hline 3NF from [16] & EIHH & NCSM [16] & HH [26] & Nature \\
\hline${ }^{3} \mathrm{H}$ & $-8.474(1)$ & $-8.473(5)$ & -8.474 & -8.48 \\
${ }^{3} \mathrm{He}$ & $-7.734(1)$ & & -7.733 & -7.72 \\
${ }^{4} \mathrm{He}$ & $-28.357(7)$ & $-28.34(2)$ & -28.37 & -28.30 \\
\hline 3NF from [17] & EIHH & NCSM [17] & Nature \\
\hline \multicolumn{2}{c}{${ }^{3} \mathrm{H}$} & $-8.472(3)$ & $-8.473(4)$ & -8.48 \\
${ }^{3} \mathrm{He}$ & $-7.727(4)$ & $-7.727(4)$ & -7.72 \\
${ }^{4} \mathrm{He}$ & $-28.507(7)$ & $-28.50(2)$ & & -28.30 \\
\hline \hline
\end{tabular}

dius: 1.432(2) fm 20 for AV18+UIX and 1.464(2) fm for $\mathrm{N}^{3} \mathrm{LO}$ plus the $\mathrm{N}^{2} \mathrm{LO}$ of [17] which is not far from the experimental value of 1.463(6) fm (obtained from the charge radius of Ref. 21] as explained in 221). Also shown in Fig. 2 is the result by Viviani et al. 18] with the AV18+UIX potential, which is indistinguishable from ours, proving the level of accuracy of contemporary fourbody calculations.

Calculational Method. Our calculations are based on the diagonalization of the Hamiltonian on a square integrable hyperspherical harmonics $(\mathrm{HH})$ basis. The HH convergence is accelerated using the Suzuki-Lee unitary transformation, which then leads to the Effective Interaction HH (EIHH) method [23,24]. The high accuracy of this approach can be inferred from the benchmark results in Ref. 25] and also here from Table I, where we present the binding energies of three- and four-body nuclei obtained from EFT potentials including 3NF. We 
agree with other methods at the $10 \mathrm{keV}$ level.

Results for $S_{\mathcal{M}}(q, \omega)$ are often obtained by discretizing the continuum, where the Hamiltonian is represented on a finite basis of square integrable functions and is then diagonalized to obtain eigenvalues $e_{\nu}$ and eigenfunctions $|\nu\rangle$. In this way one achieves an ill defined discretized representation of $S_{\mathcal{M}}(q, \omega)$. On the contrary in the Lorentz integral transform (LIT) approach 27, 28] a continuum discretization can be properly used to reach the correct continuum spectrum (for various benchmark tests of the LIT approach we refer to Ref. [28]).

In the LIT case one has

$$
\mathcal{L}_{\mathcal{M}}(q, \sigma, \Gamma)=-\frac{1}{\pi} \operatorname{Im}\left\langle 0\left|\mathcal{M}^{\dagger}(q) \frac{1}{\sigma-H+E_{0}+i \Gamma} \mathcal{M}(q)\right| 0\right\rangle,
$$

where $\Gamma$ is finite (compare with Eq. (10). It is easy to prove that $\mathcal{L}_{\mathcal{M}}(q, \sigma, \Gamma)$ is connected to $S_{\mathcal{M}}(q, \omega)$ by an integral transform with a Lorentzian kernel $K(\omega, \sigma, \Gamma)=$ $\frac{\Gamma}{\pi} \frac{1}{\left(\omega+E_{0}-\sigma\right)^{2}+\Gamma^{2}}$,

$$
\mathcal{L}_{\mathcal{M}}(q, \sigma, \Gamma)=\int d \omega K(\omega, \sigma, \Gamma) S_{\mathcal{M}}(q, \omega)
$$

Since $\Gamma$ is finite the calculation of $\mathcal{L}_{\mathcal{M}}(q, \sigma, \Gamma)$ is a boundstate like problem and thus it is legitimate to represent the Hamiltonian on a basis of square integrable functions, which then leads to the following expression:

$$
\mathcal{L}_{\mathcal{M}}(q, \sigma, \Gamma)=\frac{\Gamma}{\pi} \sum_{\nu=1}^{N} \frac{|\langle\nu|\mathcal{M}(q)| 0\rangle|^{2}}{\left(\sigma-e_{\nu}+E_{0}\right)^{2}+\Gamma^{2}} .
$$

The number of basis functions $N$ depends in our EIHH calculation on the maximal value $K_{\max }$ of the $\mathrm{HH}$ grand angular momentum quantum number $K$. Note that the set $\left(e_{\nu},|\nu\rangle\right)$ is $\Gamma$-independent, but that the convergence of $\mathcal{L}_{\mathcal{M}}$ is strongly correlated with $\Gamma$ : if $\Gamma$ is lowered a higher density of states is needed, hence $K_{\max }$ and thus $N$ have to be increased. In our present case we reached convergence of $\mathcal{L}_{\mathcal{M}}$ with $\Gamma$ as small as $5 \mathrm{MeV}$ employing more than $10^{5}$ states $|\nu\rangle$. Even if this is not sufficient to resolve the ${ }^{4} \mathrm{He}^{*}$ resonance width of $270 \mathrm{keV}$, we are nevertheless able to determine the resonance energy $E_{R}$. In fact our discrete spectrum shows as first excitation above the ${ }^{4} \mathrm{He}$ ground state a very pronounced state with strength $s_{1}(q)=|\langle 1|\mathcal{M}(q)| 0\rangle|^{2}$, thus we identify the corresponding energy $e_{1}$ with $E_{R}$. We find the following results: $E_{R}=-7.40(20) \mathrm{MeV}(\mathrm{AV} 18+\mathrm{UIX})$ and $E_{R}=-7.50(30)$ $\mathrm{MeV}\left(\mathrm{N}^{3} \mathrm{LO}+\mathrm{N}^{2} \mathrm{LO}\right)$. Note that error estimates are made by studying the EIHH convergence, i.e. the $K_{\max }$ dependence of $E_{R}$ and that the $E_{R}$ value for $\mathrm{N}^{3} \mathrm{LO}+\mathrm{N}^{2} \mathrm{LO}$ is obtained extrapolating to higher $K_{\max }$ with an exponential ansatz $E\left(K_{\max }\right)=E^{\infty}+a e^{-b K_{\max }}$ as in $[33$.

In general one obtains the full strength $S(q, \omega)$ from the inversion of a converged LIT, but one has to be aware that structures much smaller than $\Gamma$ cannot be resolved and thus a regularization procedure has to be used [29, 30]. Our standard inversion method consists in an expansion of the response on a set of $I$ continuous
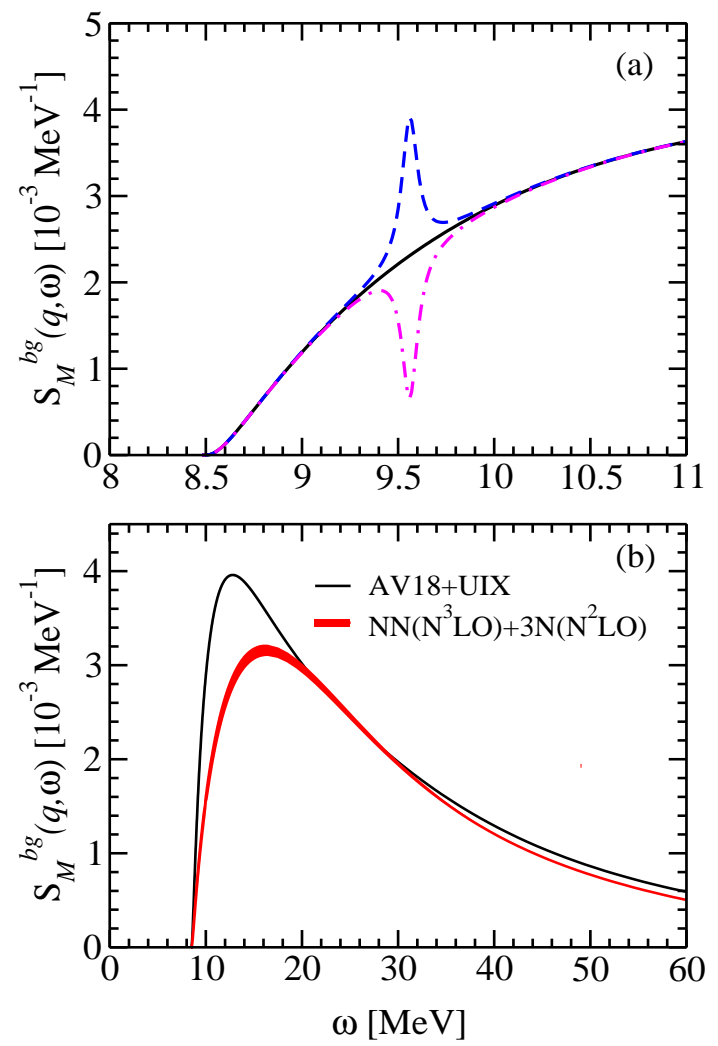

FIG. 3. (Color online) (a) $\mathcal{S}_{\mathcal{M}}^{b g}(q, \omega)$ at $q=1.5 \mathrm{fm}^{-1}$ for AV18+UIX obtained with different values of $f_{R}$ (see text): $f_{R}=\left|F_{\mathcal{M}}(q)\right|^{2}$ (full line), $f_{R}=0.99\left|F_{\mathcal{M}}(q)\right|^{2}$ (dashed line); $f_{R}=1.01\left|F_{\mathcal{M}}(q)\right|^{2}$ (dot-dashed line); (b) non-resonant background contribution $\mathcal{S}_{\mathcal{M}}^{b g}(q, \omega)$ : AV18+UIX (full line); $\mathrm{N}^{2} \mathrm{LO}+\mathrm{N}^{3} \mathrm{LO}$ (red band).

functions and in fitting the calculated $\mathcal{L}_{\mathcal{M}}(q, \sigma, \Gamma)$ on the corresponding linear combinations of the transformed basis functions [30]. Note that the regularization consists in the fact that $I$ should not become so large that structures much smaller than $\Gamma$ appear in the inversion result. We implement many different basis and choose the best fit for a given $I$ (for example we use basis sets of the form $E^{\beta} \exp (-\alpha E / i)$ with $i=1, \ldots, I$ and different $\alpha$ values; $\beta$ is known from threshold behavior of the response, e.g. $\beta=1 / 2$ for $\left.S_{\mathcal{M}}\right)$. For example, such a calculation has been made in Refs. 31, 32 for the full ${ }^{4} \mathrm{He}$ longitudinal response beyond the ${ }^{4} \mathrm{He}^{*}$ resonance.

In the presence of a narrow resonance, as in our case, an explicit resonance should be added to the basis, e.g., a Lorentzian with free parameters $\gamma$ and $\omega_{R}$ : $\left[\left(\omega-\omega_{R}\right)^{2}+(\gamma / 2)^{2}\right]^{-1}$. If the LIT is determined with a sufficiently small $\Gamma$, then position, width, and strength of the resonance can be determined in the inversion [34]. If we proceed in this way in our present case, imposing $\omega_{R}=E_{R}$, we obtain the best fits with $\gamma \rightarrow 0$. This reflects the absence of states $|\nu\rangle$ in the vicinity of $E_{R}$. We can nonetheless determine, besides $E_{R}$, also the resonance strength $f_{R}(q)$. For this purpose we note that the above defined strength $s_{1}(q)$ is equal to the sum of $f_{R}(q)$ 
TABLE II. $\left|F_{\text {el }}\right|$ and $s_{1}=|\langle 1|\mathcal{M}(q)| 0\rangle|^{2}$ for $q=1.01 \mathrm{fm}^{-1}$ as a function of the grandangular momentum $K_{\max }$ with $\mathrm{N}^{3} \mathrm{LO}+\mathrm{N}^{2} \mathrm{LO}$ [17].

\begin{tabular}{ccccc}
\hline \hline$K_{\max }$ & 12 & 14 & 16 & 18 \\
\hline$\left|F_{\mathrm{el}}\right|$ & 0.6248 & 0.6244 & 0.6242 & 0.6241 \\
$10^{4} s_{1} / 4 \pi Z^{2}$ & 4.59 & 4.75 & 4.85 & 4.87 \\
\hline
\end{tabular}

and a background contribution. Thus, formally, we can separate the resonance contribution from $\mathcal{L}_{\mathcal{M}}$ :

$$
\mathcal{L}_{\mathcal{M}}^{b g}(q, \sigma, \Gamma)=\mathcal{L}_{\mathcal{M}}(q, \sigma, \Gamma)-\frac{\Gamma}{\pi} \frac{f_{R}(q)}{\left(\sigma-E_{R}+E_{0}\right)^{2}+\Gamma^{2}} .
$$

Now we proceed as follows. We assume a value for $f_{R}(q)$ and allow a basis function of Lorentzian shape centered at $E_{R}$ with $\gamma=100 \mathrm{keV}$ in the inversion. If the trial value for $f_{R}(q)$ is too small/large one finds an inversion with a positive/negative resonant structure. The case where this vanishes corresponds to the correct value of the transition form factor $\left|F_{\mathcal{M}}(q)\right|^{2}=f_{R}(q) / Z^{2}$ and the inversion result is just $S_{\mathcal{M}}^{\mathrm{bg}}(q, \omega)$ (see Fig. 3). We would like to emphasize that the results are almost $\gamma$ independent so long $\gamma$ remains small enough $(0<\gamma \leq$ $200 \mathrm{keV}$ ) that the Lorentzian approximates sufficiently well a $\delta$-function. For the AV18+UIX potential the relative size of the background reduction, about $8 \%$, is roughly $q$ independent. For the $\mathrm{N}^{3} \mathrm{LO}+\mathrm{N}^{2} \mathrm{LO}$ interactions the reduction varies between $13 \%$ for $q=0.25 \mathrm{fm}^{-1}$ and $22 \%$ for $q=2 \mathrm{fm}^{-1}$.

In Fig. 3 b, the non-resonant monopole strength $S_{\mathcal{M}}^{\mathrm{bg}}$ is shown on a larger energy range, into the far continuum region. One sees quite a difference between the results with the EFT and the AV18+UIX forces. The former leads to a lower low-energy peak and tail than the latter. These results show the power of the LIT approach, which enables one to calculate the strength in the far four-body continuum by reducing a scattering-state problem to a bound-state problem in a rigorous way.

Analysis of the Results. The main findings of this Letter are the dramatic sensitivity of $F_{\mathcal{M}}(q)$ to the nuclear Hamiltonian and the large deviations of realistic calculations from the available experimental data. Even though one can contemplate the possibility of systematic experimental errors, the fact that the experimental results of Fig. 1 correspond to three different sets of data, makes it less likely. Thus we will now list possible sources for theoretical uncertainties.

(i) Is our EIHH expansion sufficiently convergent? As shown in Table Ifor a $q$-value of $1.01 \mathrm{fm}^{-1}$, we find an excellent convergence for both $F_{\mathrm{el}}$ and $s_{1}$.

(ii) Are there relevant two-body corrections to the onebody operator of Eq. (2)? Such corrections are of rela- tivistic order and appear also in EFT only at 4th order [35] (also for $F_{\mathrm{el}}(q)$ such two-body terms are negligible below $q=2 \mathrm{fm}^{-1}[18]$ ).

(iii) Can additional 3NF terms change the picture? This is not excluded, however we notice that the $3 \mathrm{NF}$ effect at $\mathrm{N}^{2} \mathrm{LO}$ on $F_{\mathcal{M}}(q)$ is rather mild (about $10 \%$ ).

(iv) Does the improper theoretical resonance position $E_{R}$ affect the $F_{\mathcal{M}}(q)$ result? Both our potential models $\left(\mathrm{AV} 18+\mathrm{UIX}, \mathrm{N}^{3} \mathrm{LO}+\mathrm{N}^{2} \mathrm{LO}\right)$ overestimate $E_{R}$ by almost the same amount (about $700 \mathrm{keV}$ ), but still lead to quite different transition form factors. On the other hand, the simplified force model used by Hiyama et al. [10] reproduces the correct $E_{R}$ within $100 \mathrm{keV}$, and also leads to a much better description of $F_{\mathcal{M}}(q)$. One can envisage a correlation between the ability of a model to reproduce $E_{R}$ and $F_{\mathcal{M}}$. In fact, if one considers that $F_{\mathcal{M}}$ is the Fourier transform of the transition density from ${ }^{4} \mathrm{He}$ to ${ }^{4} \mathrm{He}^{*}$, one can imagine that small differences in $E_{R}$ are reflected in the resonant wave functions and yield larger differences in the transition density. Similar conclusions have been drawn in Ref. [36] in the study of $p-{ }^{3} \mathrm{H}$ scattering. However, the resonant behavior of the nuclear scattering amplitude is barely visible in the data, in contrast to the electromagnetic probe that amplifies the resonance signal considerably (see Fig. 1 of Ref. [12]).

Conclusions. We have calculated the isoscalar monopole ${ }^{4} \mathrm{He} \longrightarrow{ }^{4} \mathrm{He}^{*}$ transition form factor $F_{\mathcal{M}}(q)$ with realistic nuclear forces $\left(\mathrm{N}^{3} \mathrm{LO}+\mathrm{N}^{2} \mathrm{LO}, \mathrm{AV} 18+\mathrm{UIX}\right)$. Unexpectedly the results are strongly dependent on the Hamiltonian. Therefore this observable is ideal for testing nuclear Hamiltonians. As surprising as the large potential model dependence, is the fact that our $F_{\mathcal{M}}$ results are at variance with the experimental data, particularly large differences are found in the case of the chiral forces. It is very unlikely that corrections to the isoscalar monopole operator can lead to large effects. In order to clarify the situation it is highly desirable to have a further experimental confirmation of the existing data and in particular with increased precision. On the theory side further insight could be gained by an analysis of sum rules, transition densities, effects of D-wave components and different $3 \mathrm{NFs}$. These issues will be the subject of future studies.

We would like to thank Thomas Walcher for his helpful discussions and explanations about the experiments. We would like to thank Michele Viviani for providing us with his theoretical results for $F_{\text {el }}$. Acknowledgments of financial support are given to Natural Sciences and Engineering Research Council (NSERC) and the National Research Council of Canada, S.B., the Israel Science Foundation (Grant number 954/09), N.B., the MIUR grant PRIN-2009TWL3MX, W.L. and G.O.. We would also like to thank the INT for its hospitality during the preparation of this work (INT-PUB-12-050). 
[1] D.H. Youngblood et al., Phys. Rev. Lett. 39, 1188 (1977).

[2] O. Bohigas, A. M. Lane, and J. Martorell, Phys. Rep. 51, 267 (1979).

[3] J.P. Blaizot, Phys. Rep. 64, 171 (1980).

[4] Th. Walcher, Phys. Lett. B, 31, 442 (1970).

[5] S. Fiarman and W.E. Meyerhof, Nucl. Phys. A, 2061 (1973); D.R. Tilley, H.R. Weller, G.M. Hale, Nucl. Phys. A, 541 1-104 (1992).

[6] W. Leidemann and G. Orlandini, arXiv:1204.4617 Progr. Part. Nucl. Phys., in press.

[7] S. Weinberg, Phys. Lett. B 251, 288 (1990); C. Ordez, L. Ray, U. van Kolck, Phys. Rev. Lett. 72, 1982 (1994); E. Epelbaum, W. Glöckle, U.-G. Meißner, Nucl. Phys. A 637, 107 (1998).

[8] A. Kievsky, Few-Body Syst. 50, 69 (2011).

[9] S. Galster et al., Nucl. Phys. B 32, 221 (1971).

[10] E. Hiyama, B.F. Gibson, and M. Kamimura, Phys. Rev. C 70, 031001 (R) (2004).

[11] R.F. Frosch et al., Phys. Lett. 19, 155 (1965); Nucl. Phys. A, 110, 657 (1968).

[12] G. Köbschall et al., Nucl. Phys. A, 405, 648 (1983).

[13] R.B. Wiringa, V.G.J. Stoks, and R. Schiavilla, Phys. Rev. C 51, 38 (1995).

[14] B.S. Pudliner et al., Phys. Rev. Lett. 74, 4396 (1995).

[15] D.R. Entem and R. Machleidt, Phys. Rev. C 68, 041001 (2003).

[16] P. Navrátil, Few Body Syst., 41, 117 (2007).

[17] D. Gazit, S. Quaglioni, and P. Navrátil, Phys. Rev. Lett. 103, 102502 (2009).
[18] M. Viviani et al., Phys. Rev. Lett. 99, 112002 (2007).

[19] R.F. Frosch et al., Phys. Rev. 160, 874 (1967).

[20] D. Gazit et al., Phys. Rev. Lett. 96112301 (2006).

[21] I. Sick, Phys. Rev. C 77, 041302R (2008).

[22] The charge radius $r_{\mathrm{C}}$ is converted to a point-proton radius $r_{\mathrm{pp}}$ by $r_{\mathrm{pp}}^{2}=r_{\mathrm{c}}^{2}-R_{p}^{2}-(N / Z) \cdot R_{n}^{2}-0.033 \mathrm{fm}^{2}$ where $R_{p}=0.877(7)$ is the proton finite size, $R_{n}^{2}=-0.1161(22)$ is the neutron finite size correction and the last term is a relativistic correction.

[23] N. Barnea, W. Leidemann, and G. Orlandini, Phys. Rev. C 61, 054001 (2000); Nucl. Phys. A 693, 565 (2001).

[24] N. Barnea and A. Novoselsky, Phys. Rev. A 57, 48 (1998); Ann. Phys. (N.Y.) 256, 192 (1997).

[25] H. Kamada et al., Phys. Rev. C 64, 044001 (2001).

[26] A. Kievsky et al., J. Phys. G 35, 063101 (2008).

[27] V. D. Efros, W. Leidemann, and G. Orlandini, Phys. Lett. B 338, 130 (1994).

[28] V. D. Efros et al., J. Phys. G, 34, R459 (2007).

[29] A.N. Tikonov and V.Y. Arsenin, Solutions of Ill-Posed Problems, Washington, DC: V H Winston and Sons, 1977.

[30] D. Andreasi et al., Eur. Phys. J. A 24, 361 (2005).

[31] S. Bacca et al., Phys. Rev. C 80, 064001 (2009).

[32] S. Bacca et al., Phys. Rev. C 76, 014003 (2007).

[33] S. Bacca et al., Eur. Phys. J. A 42553 (2009).

[34] W. Leidemann, Few-Body Syst. 42, 139 (2008).

[35] T.-S. Park et al., Phys. Rev. C 67, 055206 (2003).

[36] R. Lazauskas, Phys. Rev. C 79, 054007 (2009). 